\title{
Design of a Tandem Compressor for the Electrically- Driven Turbocharger of a Hybrid City Car ${ }^{\dagger}$
}

\author{
Nicolò Cuturi * and Enrico Sciubba \\ Department of Mechanical and Aerospace Engineering, University of Roma Sapienza, 00185 Roma, Italy; \\ nicolo.cuturi@gmail.com, enrico.sciubba@uniroma1.it \\ * Correspondence: nicolo.cuturi@gmail.com \\ + Presented at the First World Energies Forum, 14 September-5 October 2020; Available online: \\ https://wef.sciforum.net/.
}

Published: 12 September 2020

\begin{abstract}
Within a broader national project aimed at the hybridization of a standard city car (the 998 cc Mitsubishi-derived gasoline engine of the Smart W451), our team tackled the problem of improving the supercharger performance and response. The design concept is that of eliminating the mechanical connection between the compressor and the turbine. It turned out that it is also possible to modify both components to extract extra power from the engine and to use it to recharge the battery pack. First, the initial configuration was analyzed on the basis of the design data provided by the manufacturer. Then, a preliminary performance assessment of the turbocharged engine allowed us to identify three "typical" operating points that could be used to properly redesign the turbomachinery. It was decided to maintain the radial configuration for both turbine and compressor, but to redesign the latter by adding an inducer. For the turbine, only minor modifications to the NGV and rotor blades shape were deemed necessary. Fully 3-D CFD simulations of the rotating machines were performed to assess their performance at three operating points: the kick-in point of the original turbo $(2000 \mathrm{rpm})$, the maximum power regime (5500 rpm) and an intermediate point (3500 rpm) close to the minimum specific fuel consumption (SFC) for the original engine. The results presented in this paper demonstrate that the efficiency of the compressor is noticeably improved for steady operation at all three operating points, and that its choking characteristics have been improved, while its surge line has not been appreciably affected. The net energy recovery was also calculated, and demonstrated interesting returns in terms of storable energy in the battery pack.
\end{abstract}

Keywords: hybrid engine; turbocharging; radial compressor CFD; tandem compressor CFD; entropy generation maps

\section{Introduction}

The increasing concern about $\mathrm{CO}_{2}$ emissions has led to stricter regulations of the acceptable quality of the exhaust gases of internal combustion engines (ICE in the following). With regard to gasoline-fueled ICE, the automotive industry response has taken three directions: the downsizing of existing engines [1,2], the introduction of hybrid propulsion systems [3], and the design of all-electric vehicles. The three solutions are not equivalent in terms of feasibility, cost and time-to-market, and only the first one is based on sufficiently mature technology to be immediately implemented without substantial modifications to the production and assembly lines. Within this scenario, the Italian Ministry of Research launched a series of R\&D projects aimed at a better definition of short-term modifications to existing gasoline ICEs.

Since passenger vehicles powered by downsized engines are not palatable for the general European customer (who "demands" power), an increase in the ICE power density is necessary: 
turbocharging is an industrially mature measure whose appearance in large-series commercial power units dates back to the 1970s [4]. It has been continuously improved in the last decade, and the advance in electronic control units has led to its adoption on large series commercial vehicles.

In gasoline-fueled ICE, most turbocharger units today are back-to-back, radial/radial compressor/turbine assemblies (Figure 1): the exhaust gases from the engine power the turbine that in turn powers the compressor (other solutions adopted in commercial vehicles include the use of volumetric compressors, but they are not investigated here). A schematic representation of the process and of the subsumed ideal and real (indicated) cycle is shown in Figure 2 [5].

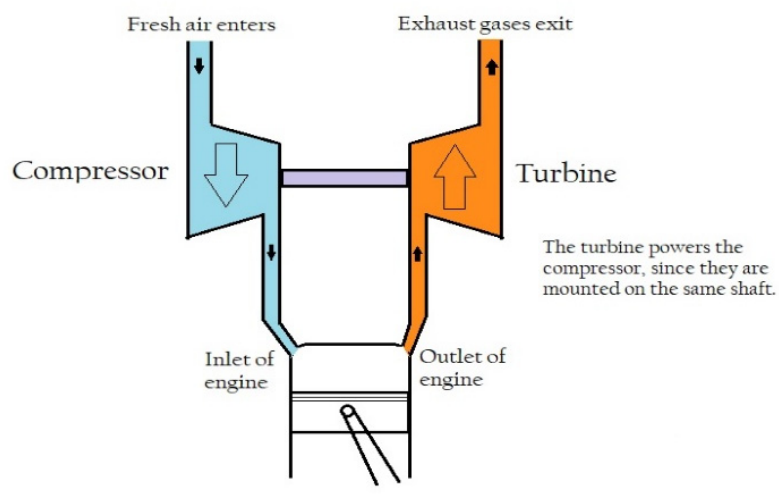

Figure 1. Standard turbocharger assembly.

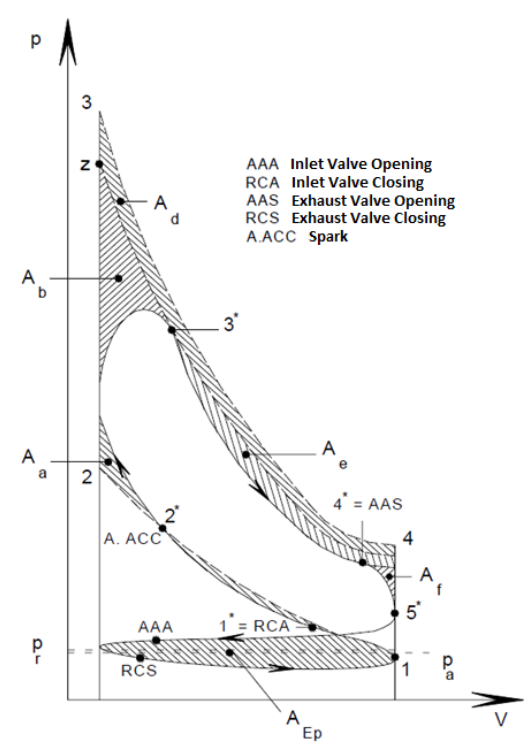

Figure 2. The supercharged indicated cycle, with direct comparison to the ideal Otto cycle.

Even neglecting the unavoidable flow fluctuations intrinsic in reciprocating piston engines, "normal" engine operating curves are not stationary but semi-periodically changing over time, so that the mass flowrate of both the intake air (point 1 in Figure 2) processed by the compressor and that at engine exhaust/turbine entry (point $4-5^{\prime}$ in the same figure) vary continuously, as well as the exhaust temperature and pressure (and, strictly speaking, also the exhaust gas composition). Since the operating curves of the two components have substantially different characteristics (Figures 3 and 4), in modern turbocharged engines a dedicated electronic control unit (ECU) ensures a proper matching, either by limiting the mass flowrate through the turbine via the controlled venting of a portion of the exhaust gases directly into the discharge plenum through a valve called "waste gate", or by modifying the stagger of the turbine NGV, or both. The most popular configuration today consists of a small and fast radial compressor with fixed radial blades and a non-vaned diffuser and of a radial turbine with fixed NGV: only a few units are equipped with variable geometry NGV. A 
perusal of the most recent literature on radial compressors shows though that a "tandem rotor", in which the incoming air flows first through a specifically designed axial "inducer" before entering the radial portion of the blades, seems to offer an extended operational field, increased efficiency and compression ratio [6-9]. One of the aims of this study is to develop and compare an inducer-bladed (Tandem) version of a compressor starting from a conventional impeller based on the Garrett GT12. The inducer is treated as an axial compressor cascade and its basic design is developed with the standard mono-dimensional turbomachinery theory. Both geometries are investigated by a series of CFD simulations performed with a commercial software (ANSYS-CFX).

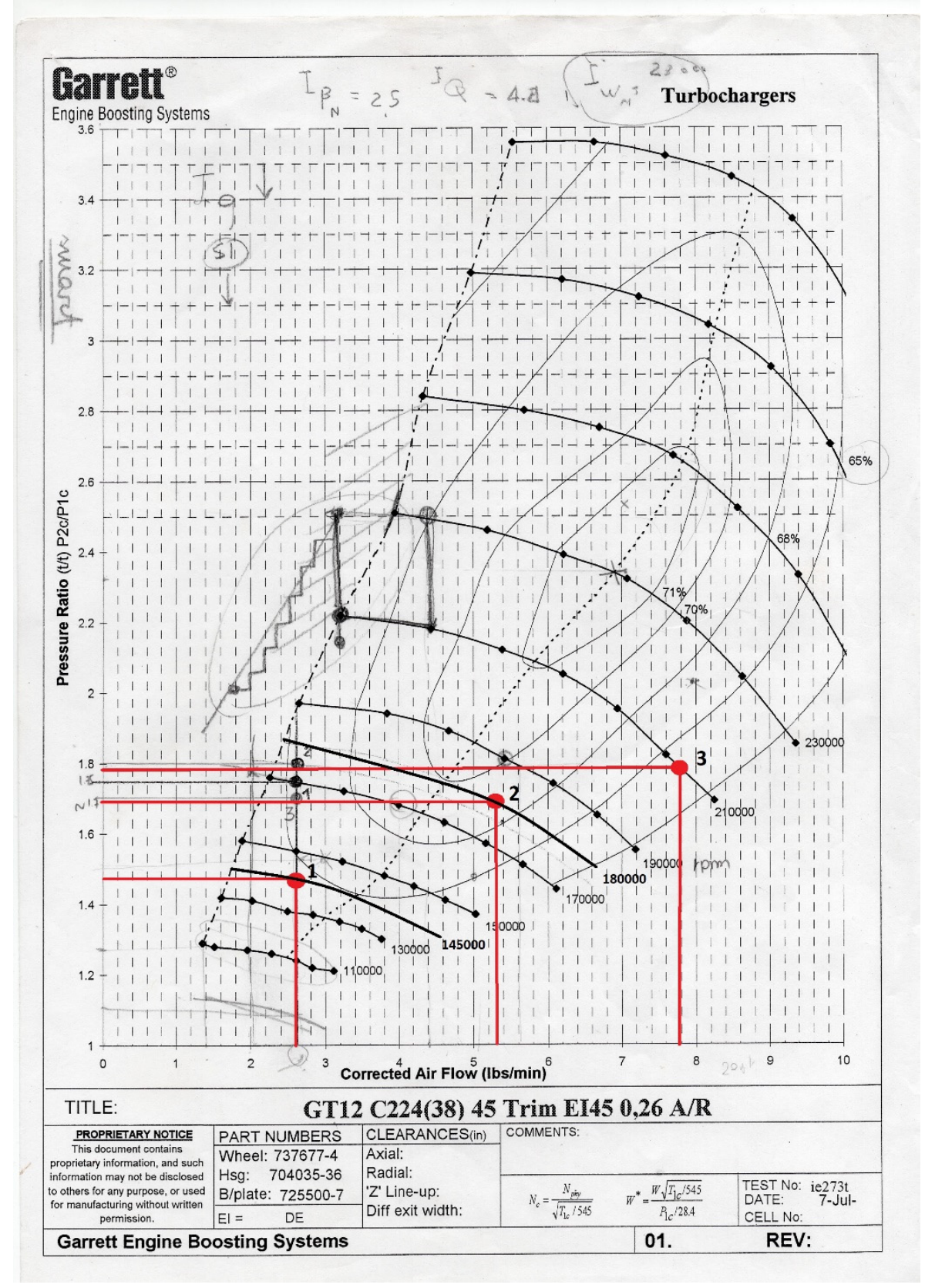

Figure 3. Garrett GT1238 compressor map. 


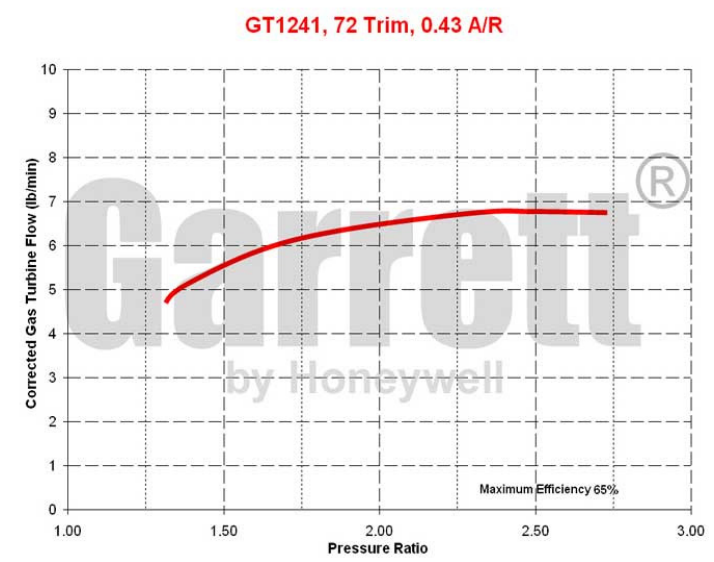

Figure 4. Garrett GT1238 turbine map.

Since it is known that the two parameters that most influence performance are the inducer/exducer axial displacement and clock, the tandem impeller geometry has undergone a design of experiment (DOE) campaign in order to optimize both the performance and range. The final "optimal" compressor maps are reported, and the ensuing flow fields are analyzed as of their respective entropy generation maps to gain a better insight into the boundary layer interaction between inducer and exducer. The analysis has been performed at three operative points representative of realistic engine conditions.

Both the design of the compressor, discussed here in detail, and that of the turbine, the subject of a related study [10], have been finalized on the basis of an initial system performance study (engine + turbocharger + auxiliaries), and the components will be built and assembled on the original ICE for bench testing.

\section{The Original Turbocharged Engine}

The engine for which the new turbocharger is being designed is the three cylinder, $1.0 \mathrm{~L}$ ( $998 \mathrm{cc}$ ) gasoline engine W451, originally produced by Mitsubishi and modified by Mercedes for the Smart ForTwo. In this very compact packaging (Figure 5), the turbocharger unit is mounted in a standard fashion, with no intercooling and with a direct mechanical coupling between the two rotors (i.e., they rotate at the same speed and a compromise has been struck between the optimal map of the turbine and that of the compressor, so that neither machine is operating on its optimal curve).

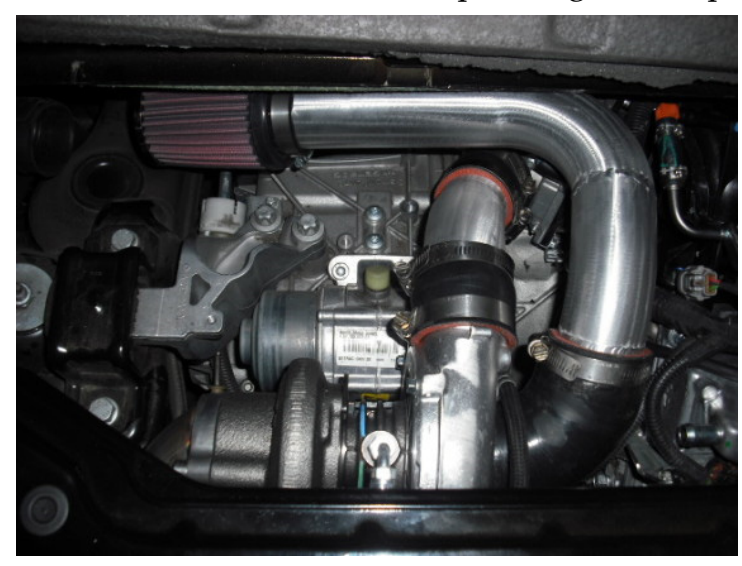

Figure 5. The turbocharged W451, 998 cc, $62 \mathrm{~kW}$ engine.

\section{Rationale of the Proposed Modifications}

At the system level, the existing configuration can be improved by adopting what is known as the "hybrid turbo compound" (Figure 6): the mechanical link between compressor and turbine is 
eliminated and each machine operates along its "optimal" curve. The compressor is powered by an electrical motor fed by the battery pack of the hybrid vehicle, and the turbine is coupled to an electrical generator that feeds the same battery pack. In this way, the excess power can be directly recovered by storing it in the form of electrical energy: this solution is particularly advantageous in a hybrid propulsion system in which additional kinetic-to-electrical energy recovery is enforced via a KERS. A properly designed electrical control unit, together with a specific engine tuning, results in the elimination of the waste gate valve, increasing the propulsion efficiency under road conditions. As a direct consequence of the mechanical separation of the two rotors, substantial savings in fuel economy are likely to emerge (less energy extracted from the battery per unit of energy delivered to the wheels).

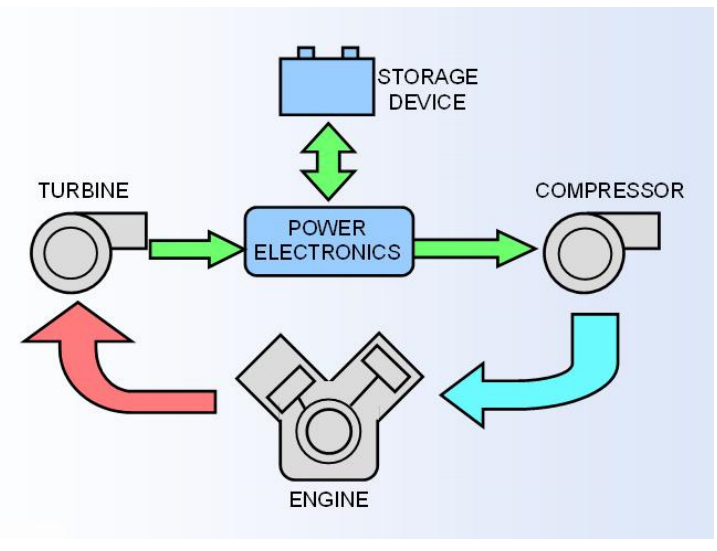

Figure 6. Hybrid turbo-compound concept.

\section{The Modified Radial Compressor: Geometry and Computational Mesh}

\subsection{The Original GT12 Compressor Geometry}

To validate our results, a CFD simulation was performed on the original GT12 compressor geometry, shown in Figure 7, left. The steps adopted in the construction are outlined here below.

1. Since the original drawings are proprietary, images and samples of existing rotors were examined to extract representative quantitative information (diameters, angles, curvatures, blade thickness, etc.) sufficient to generate a proper geometrical model and to digitalize the obtained geometries using Vista CCD;

2. The virtual design thus obtained was lengthened by 1.5 diameters of the inlet eye $(30 \mathrm{~mm})$ on the intake side, so that the numerical code may "adjust" the flow quantities from the inlet b.c. to the blade leading edge. Similarly, the outlet was lengthened by $10 \%$ of the blade tip diameter ( 2 $\mathrm{mm})$;

3. The blade profile was generated as a cubic spline. The incidence $\beta$ was specified from the geometrical drawing, and a blade overlap of $\theta=60^{\circ}$ was imposed. The splitter was set to begin at mid channel length. The blade maximum thickness is $1.3 \%$ of the chord of the blade and clearance is set to $2 \%$ of blade span.

4. Thanks to the axisymmetric geometry of the impeller, the computational domain consists of a $60^{\circ}$ solid slice of the entire impeller and includes one main blade and one splitter.

The final values of the analytical design for the conventional impeller were compared with the available data for the Garrett GT12 and were found to agree with exceptional accuracy [11]. 


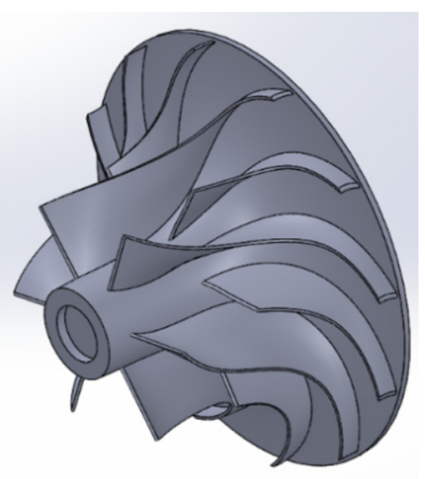

$\mathbf{L}$

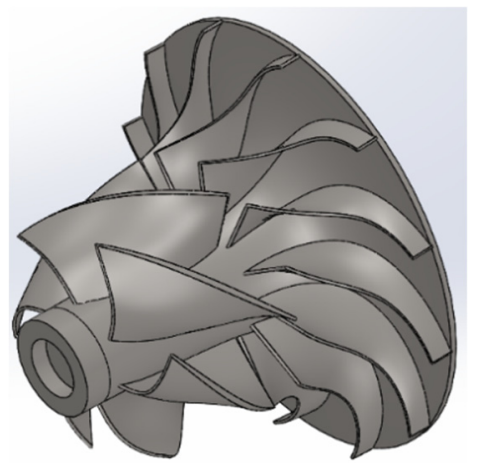

$\mathbf{R}$

Figure 7. The original GT 12 impeller (Left) and the new tandem geometry (Right).

\subsection{The Tandem Impeller Geometry}

The tandem version of the GT12 impeller has been designed with the same solid modelling tools described above, but the following modifications were introduced:

1. More accurate leading and trailing edge profiles were added to the inducer blade;

2. Both the inducer chord and stagger varied spanwise;

3. The inducer maximum thickness was set to $3.5 \%$ of chord and located at mid chord;

4. The exducer is obtained by trimming the original centrifugal blades until they reach the same chord length as the splitter blades: in practice, we are thus dealing with a 12-blade radial rotor.

\subsection{The Diffuser}

Both the original GT12 turbocharger unit and the new tandem compressor are equipped with a vaneless diffuser whose axial span is equal to the blade thickness at rotor exit. The radial extension of the diffuser is the same for both units, and the computational domain was extended by 1.5 diameters downstream. The mixing plane method was used to match the rotor and diffuser at the interface.

\section{Performance Comparisons}

The original Garrett compressor was simulated with ANSYS-CFX at steady state, with no inlet preswirl and air conditions as in Table 1: the results were found to closely agree with the available performance data [11].

Table 1. Air inlet conditions.

\begin{tabular}{ccc}
\hline Inlet Pressure $\left(\mathrm{p}_{1}\right)$ & $\mathrm{Pa}$ & 101000 \\
\hline Inlet temperature $\left(\mathrm{T}_{1}\right)$ & $\mathrm{K}$ & 298 \\
\hline Intake mass flow rate, point 1 & {$[\mathrm{~kg} / \mathrm{s}]$} & 0.021 \\
\hline Intake mass flow rate, point 2 & {$[\mathrm{~kg} / \mathrm{s}]$} & 0.042 \\
\hline Intake mass flow rate, point 3 & {$[\mathrm{~kg} / \mathrm{s}]$} & 0.062 \\
\hline Corrected mass flow, point 1 & {$[\mathrm{lb} / \mathrm{min}]$} & 2.7 \\
\hline Corrected mass flow, point 2 & {$[\mathrm{lb} / \mathrm{min}]$} & 5.2 \\
\hline Corrected mass flow, point 3 & {$[\mathrm{lb} / \mathrm{min}]$} & 7.8 \\
\hline
\end{tabular}

\subsection{The Tandem Compressor}

In order to make a proper comparison, the initial design point (DP) of the tandem rotor is set equal to that of the traditional design: maximum isentropic efficiency 0.71 and $100 \%$ revolution speed at 230,000 rpm. The reduced mass flow rate at DP is imposed by the compressor operational map at $0.055 \mathrm{~kg} / \mathrm{s}$, and the compression ratio is 2.3 . The main geometric features are reported in Table 2. 
Table 2. Main features of the tandem compressor rotor.

\begin{tabular}{ccc}
\hline & Unit & Value \\
\hline Inducer tip diameter $\left(\mathrm{D}_{1}\right)$ & $\mathrm{mm}$ & 22.47 \\
\hline Inducer hub diameter $\left(\mathrm{D}_{1 i}\right)$ & $\mathrm{mm}$ & 8.20 \\
\hline Exducer tip diameter $\left(\mathrm{D}_{2}\right)$ & $\mathrm{mm}$ & 38.00 \\
\hline Blade tip span $(\mathrm{b})$ & $\mathrm{mm}$ & 2.38 \\
\hline $\mathrm{Z}_{\mathrm{n}}$ & - & $6+12$ \\
\hline
\end{tabular}

The inducer is treated as an axial compressor rotating solidly with the exducer, confined in the same shroud and with the same internal and external diameter as the intake of the original Garrett GT12. After a careful perusal of the available literature, its compression ratio was set to 1.2.

Our first design (Tandem A) was problematic, and some modifications were necessary: a radiusdependent solidity was imposed, with the chord increasing slightly from hub to tip. In addition, the incidence of the inducer blades was set to $2^{\circ}$ to reduce the acceleration on the leading edge suction side.

The exducer geometry of Tandem A was also modified in the course of the study, and then underwent a design of experiment (DOE) that led to the development a " $\mathrm{B}$ " and a " $\mathrm{C}$ " geometries discussed below.

The tandem configuration has a higher degree of reaction than the Garrett GT12, and thus the required pressure recovery is lower, which is beneficial for the unit efficiency. Detailed geometric and kinetic data are provided in [11]. Here, we are concerned solely with the flow field results.

A mesh sensitivity analysis was run on each configuration, the objective function being the shaft torque. Quite obviously, the results are geometry-dependent (Figure 8): the final meshes contained $1.6 \times 10^{6}$ and $1.2 \times 10^{6}$ nodes for the standard and tandem rotor, respectively. The diffuser mesh contained $1.6 \times 10^{5}$ (Garrett) and $1.2 \times 10^{5}$ (Tandem) nodes. The final rotor meshes are shown in Figure 9 .
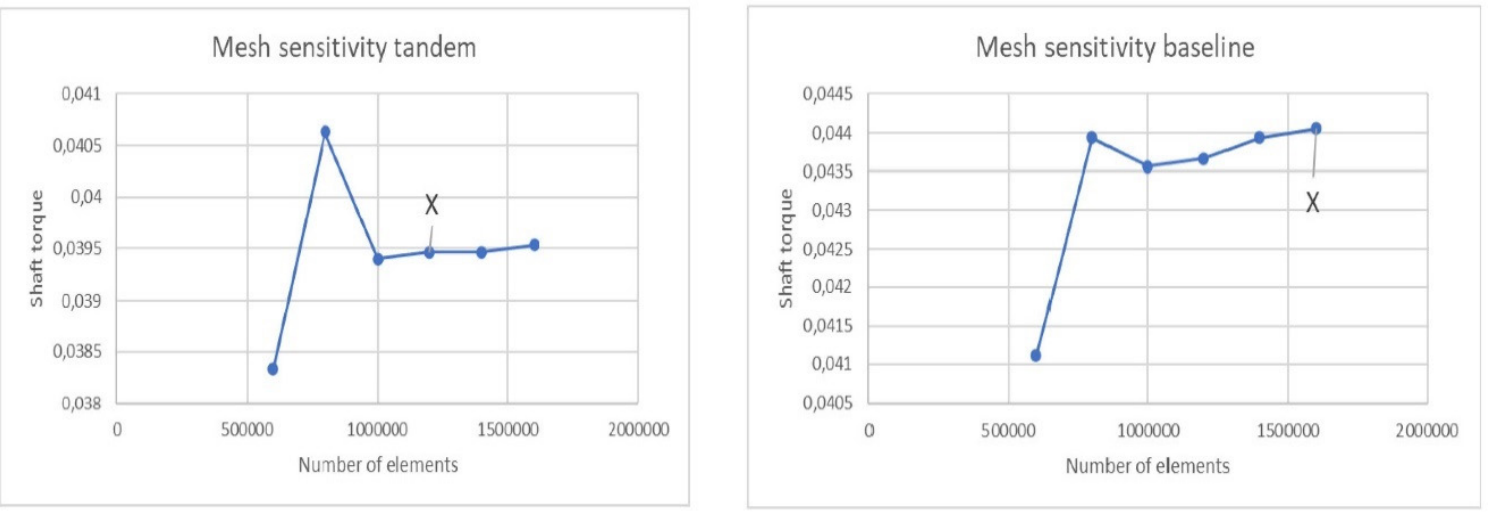

Figure 8. Mesh sensitivity for the tandem (left) and conventional (right) rotor mesh.
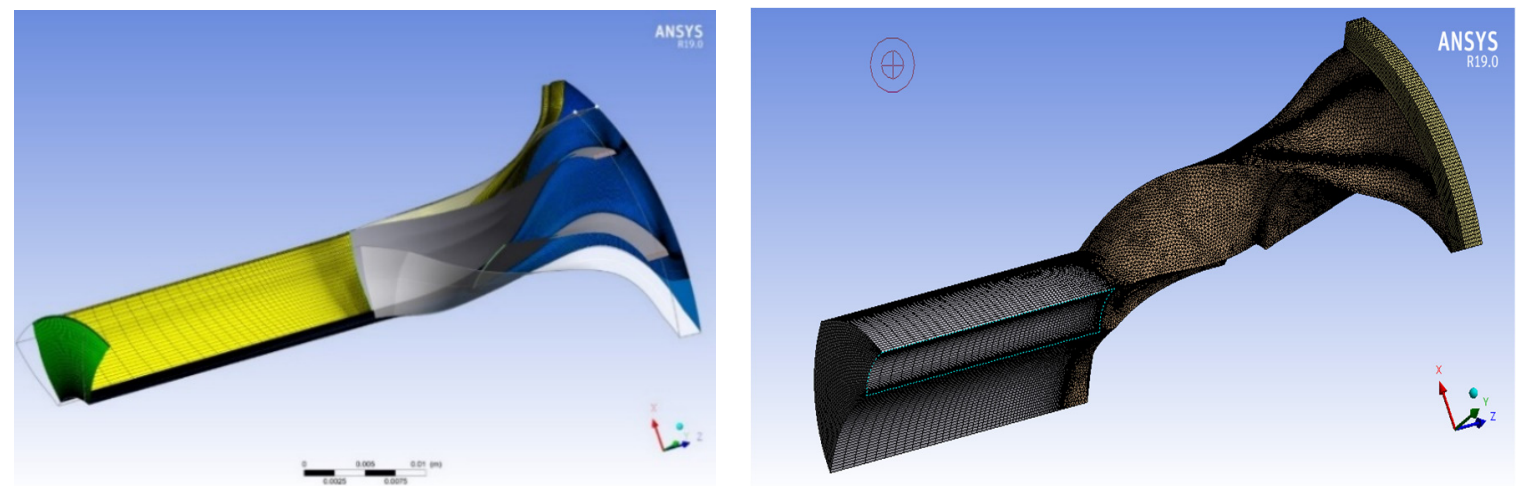

Figure 9. The final mesh for the conventional (left) and tandem compressor (right). 


\subsubsection{CFD Results}

The most interesting global result is the compressor characteristic. Figure 10 shows that the tandem A configuration:

1. displays a better efficiency than the Garrett compressor over the entire operating range (except very close to the choking line). This result was expected and well-documented in the relevant literature [7];

2. attains higher compression ratios over the entire operating range (except very close to the choking line). This result will be justified below by a detailed examination of the flowfields;

3. seems to display a better resistance to stalling (the efficiency is still high at lower mass flow ratios than its standard counterpart). This result needs a more careful analysis, and will be also discussed below, by detailed examination of the flowfields.

The relative Ma plots of Figure 11 show that the Tandem A rotor has substantially lower sonic losses at the intake, while at rotor exit the Ma profiles are similar.

The plots refer to $50 \%$ span near the choking limit [11]: under these conditions, the presence of the inducer determines a decrease in the critical mass. The Mach plots indicate the appearance of a jet flow between the pressure side of the inducer blade and the suction side of the exducer (where $\mathrm{Ma} \approx 0.8$ ): the high-speed flow flattens the boundary layer on the exducer suction side (improving work transfer in that area) but also distorts the pressure field, attracting some fluid from the suction side BL and maintaining its "jet" profile up to the rotor exit, resulting in high sonic losses.

A second characteristic of the Tandem A rotor is the mismatch between the velocity at the inducer exit and the tangent to the chord of the exducer leading edge: this results in a relatively high incidence that-at low flowrates-promotes a detachment of the BL on the pressure side of the exducer (Figures 12 and 13).

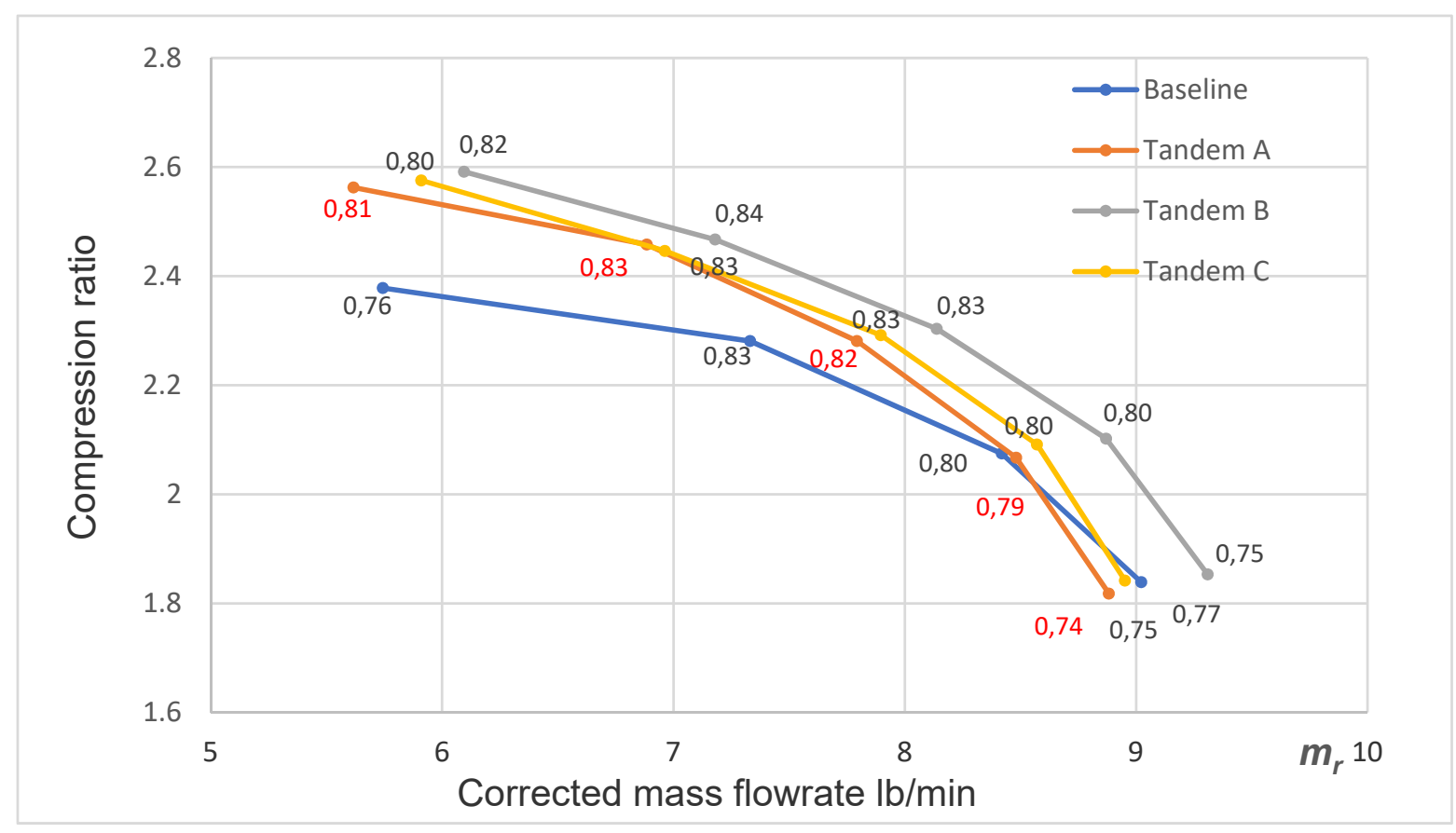

Figure 10. Characteristic curves for the conventional and tandem compressor. 

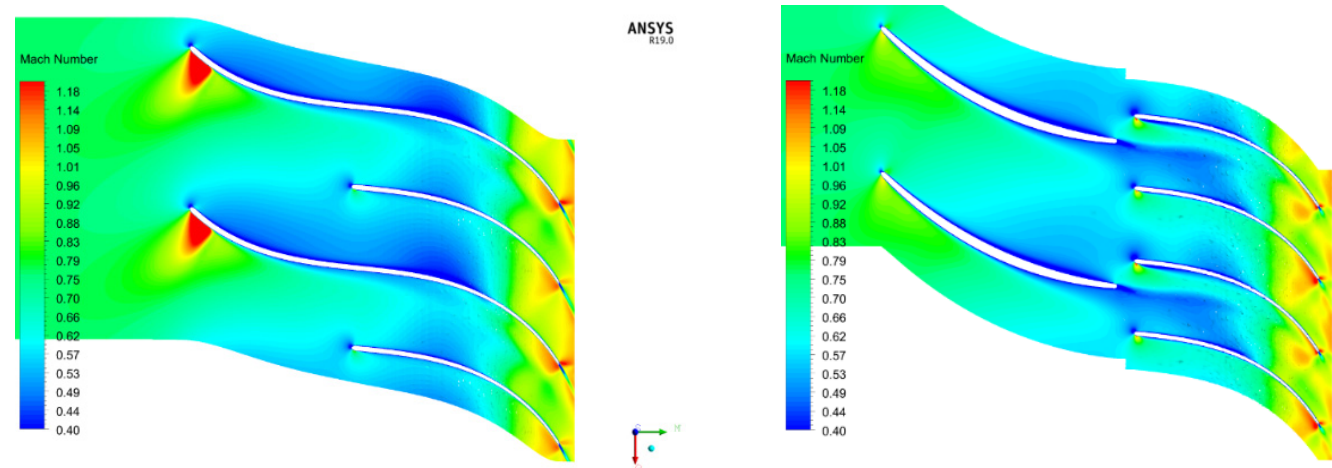

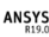

Figure 11. Relative Ma contours for the conventional (left) and tandem (right) compressor.

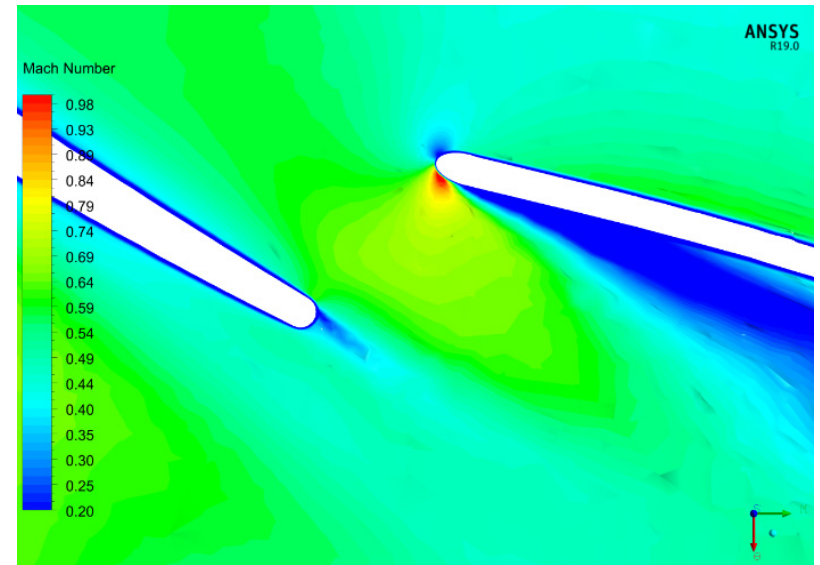

Figure 12. Excessive incidence on the exducer leading edge, $\mathrm{m}_{\mathrm{r}}=7.8(\mathrm{lb} / \mathrm{min})$.

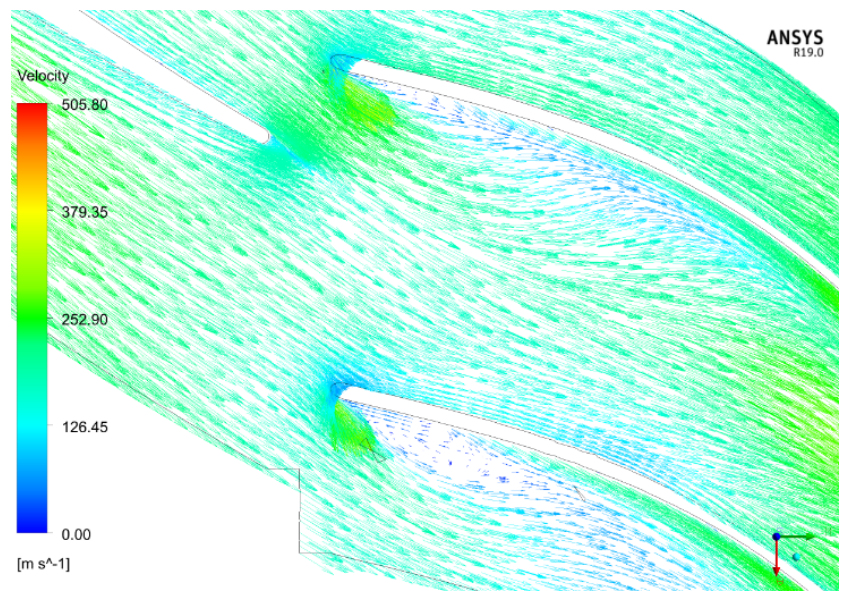

Figure 13. Recirculation on the exducer pressure side, $\mathrm{m}_{\mathrm{r}}=7.8(\mathrm{lb} / \mathrm{min})$.

Both effects are clearly due to the non-optimized design [12], and some improvements were introduced:

1. The inducer trailing edge and exducer leading edge were made radial, to enforce a radially constant angular gap between the two blades;

2. The exducer stagger has been redesigned to match the relative fluid flow leaving the inducer cascade;

3. The angular overlapping of the exducer was reduced to $40^{\circ}$, to increase the critical mass flowrate. This modified design eliminated the above negative effects (see Figures 14 and 15). 

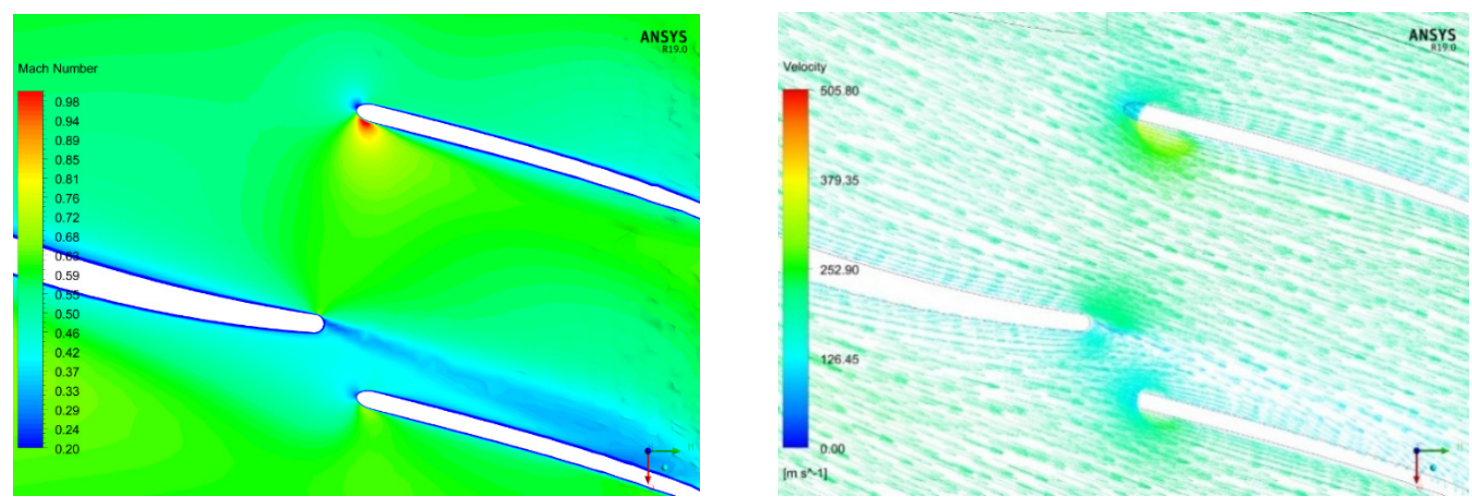

Figure 14. Effects of the incidence correction $\mathrm{m}_{\mathrm{r}}=8.8(\mathrm{lb} / \mathrm{min})$, velocity contour $(\mathbf{l e f t})$ and velocity vectors (right).

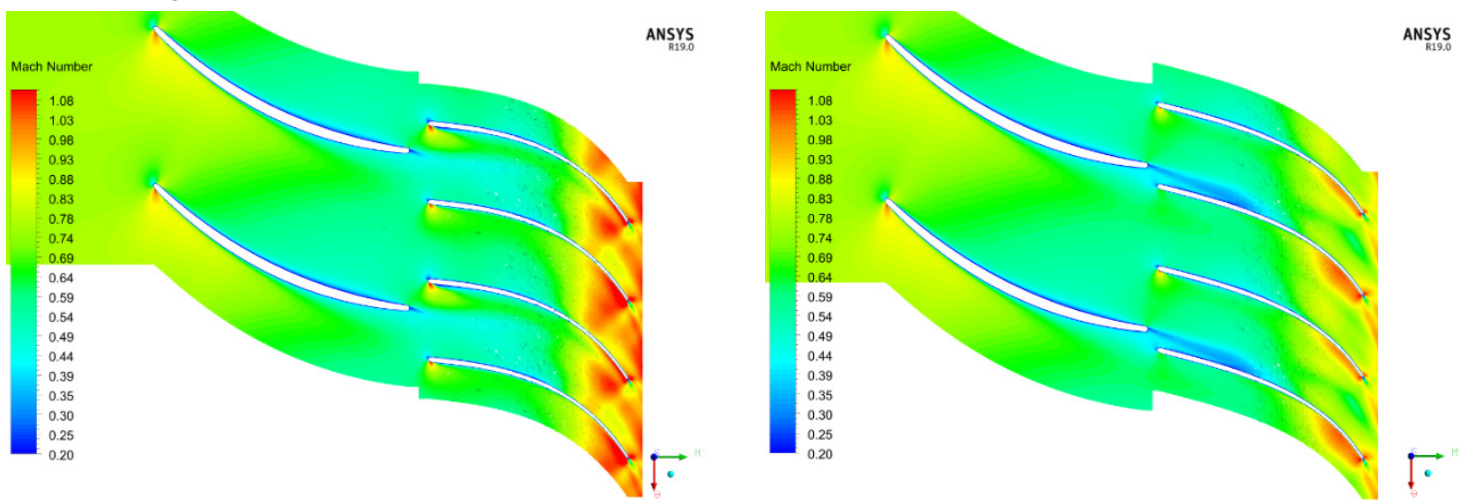

Figure 15. $\mathrm{mr}=8.8(\mathrm{lb} / \mathrm{min})$ comparison of the rotor relative Ma in tandem A (left) and Tandem B (right).

It also became clear that the two parameters that may influence the creation of a jet flow at the inducer/exducer interface are the axial clearance between the two (the larger this clearance, the more fluid "leaks" from the pressure to the suction side) and the "clock", i.e., the circumferential angle between the two blades, which is the main factor controlling the interblade jet flow (Figure 16).

Therefore, after introducing the above corrections, an experimental campaign was conducted to identify the best clock/clearance combination. Two additional configurations were compared:

- "Tandem B": $75 \%$ clock and $0.5 \mathrm{~mm}$ of axial clearance, overlap $40^{\circ}$. Obtained by a design of experiment (DOE) campaign;

- “Tandem C": $75 \%$ clock and $0.5 \mathrm{~mm}$ of axial clearance, overlap $50^{\circ}$.

The B design shows a choke margin extension, outperforming the conventional impeller, while the $C$ version (overlap increased to $50^{\circ}$ ) is slightly less efficient and just as sensitive to choke as the Garrett rotor, probably due to its higher Mach losses.
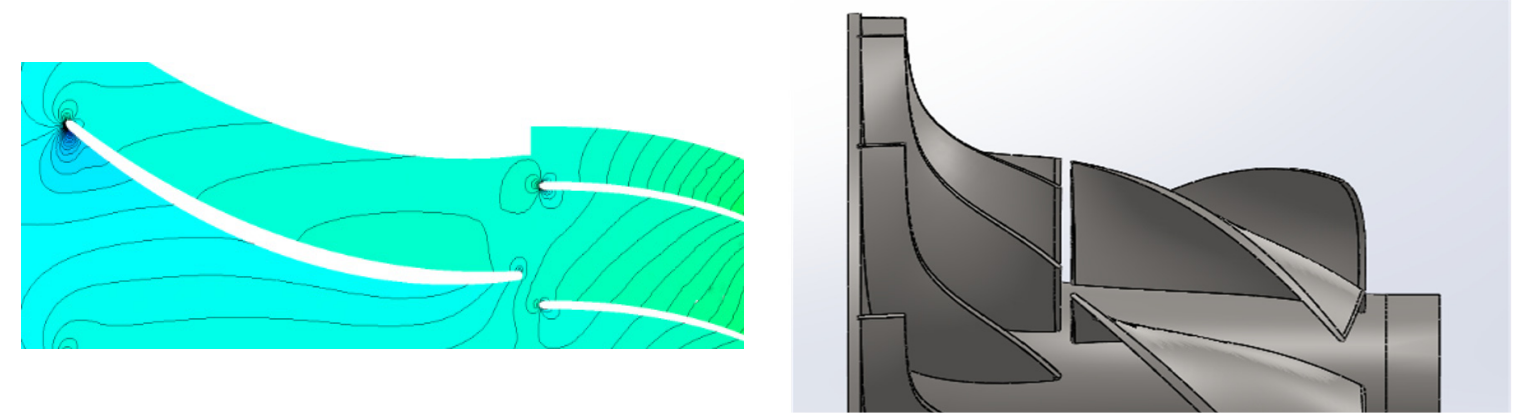

Figure 16. For the definition of "clock" (left) and "clearance" (right). 


\subsubsection{Discussion}

The most influential performance governing factor is the interaction between the inducer boundary layer (BL) and the exducer blades. In general, efficiency increases when the inducer BL. falls in the pressure side of the exducer. However, the compression ratio increases when the inducer wake "feeds" the suction side BL of the exducer, enhancing the airfoil performance.

From the analysis of the flow field emerges the fact that the optimal $75 \%$ configuration attains the highest efficiency throughout the operating range. The best balance between compression ratio and efficiency is obtained with a $0.5 \mathrm{~mm}$ clearance, that results in a particularly "clean" Mach field. The resulting final geometry is the one labeled Tandem B, which is however still affected by a noticeable amount of Mach losses at the radial exducer exit: there is no possible fix for this problem, unless a slight enlargement of the blade height at that location, to increase the passage area, is introduced. This solution has not been explored here, because it would imply a more substantial redesign of the unit.

\section{Maps of the Entropy Generation Rate}

It is instructive to examine the entropy generation rate through the blade passages, to gain some more insight into the phenomenological reasons of the performance improvement with respect to the conventional configuration. According to the Gouy-Stodola theorem, the useful work loss due to the presence of irreversible phenomena is directly proportional to the entropy generation:

$$
\dot{W}_{\text {loss }, 0}=T_{0} \dot{S}_{\text {gen }}\left[\mathrm{W} /\left(\mathrm{m}^{3}\right)\right]
$$

where $T_{0}$ is a fixed reference temperature, conveniently taken to be that of the surrounding environment. The rate of entropy generation contains two terms, a viscous and a thermal one [13]:

$$
\dot{S}_{g e n}=\frac{k \lambda}{T^{2}}(\nabla T)^{2}+\frac{\mu}{T} \Phi\left[\mathrm{W} /\left(\mathrm{m}^{3} \mathrm{~K}\right)\right]
$$

where $\lambda$ and $\mu$ are the fluid thermal conductivity and dynamic viscosity, respectively, and $\Phi$ is the viscous dissipation function, modelled in the turbulent $k-\varepsilon$ approximation adopted here and proportional to the turbulent dissipation rate. Figure 17 shows the entropy generation maps at 50\% span for the Tandem B configuration for $0^{\circ}$ and $25^{\circ}$ clock and three different values of the axial clearance.

It is apparent that increasing the clock decreases the coupling between the inducer wake and the BL developing on the pressure side of the exducer, reducing the mutual interferences and the compounding of the two dissipative effects. However, the $25 \%$ as well as the $50 \%$ clock configurations are strongly influenced by the jet flow that decreases the performance with respect to the $0 \%$ case. As for the axial clearance, while in the $0 \%$ clock an increase in the gap leads to some bleeding of the inducer BL towards the suction side, worsening the efficiency, in the $25 \%$ and $50 \%$ cases, an increased clearance increases the throat section, lowering the Mach losses.
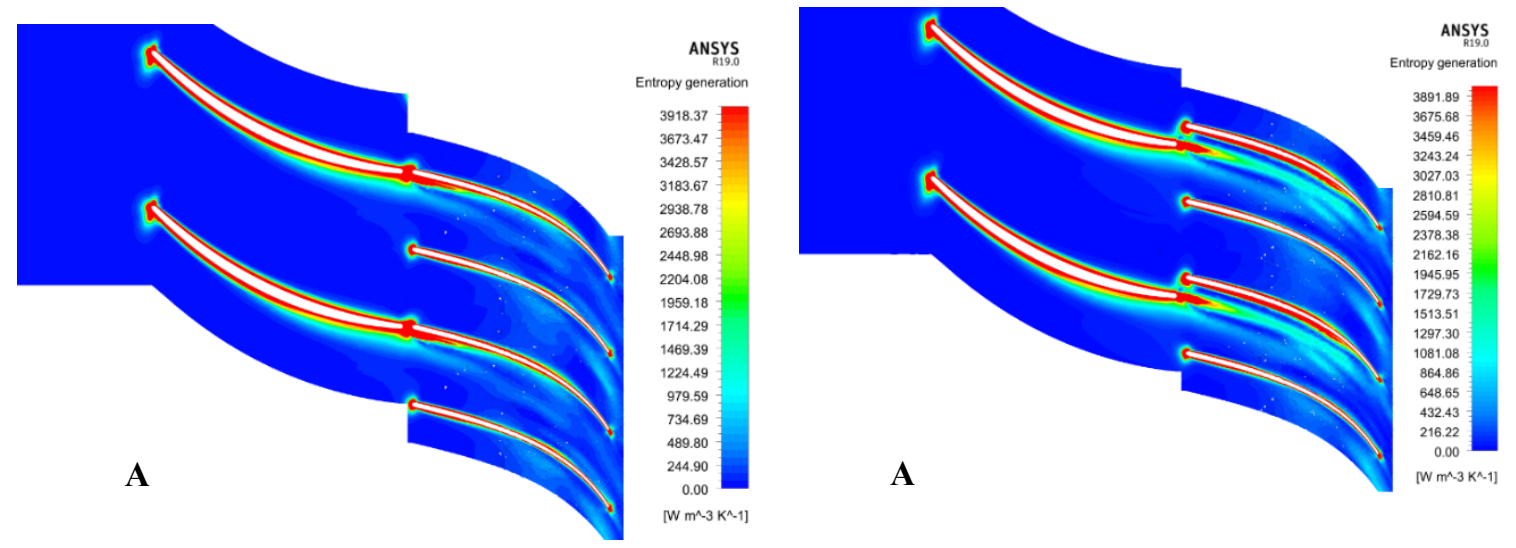

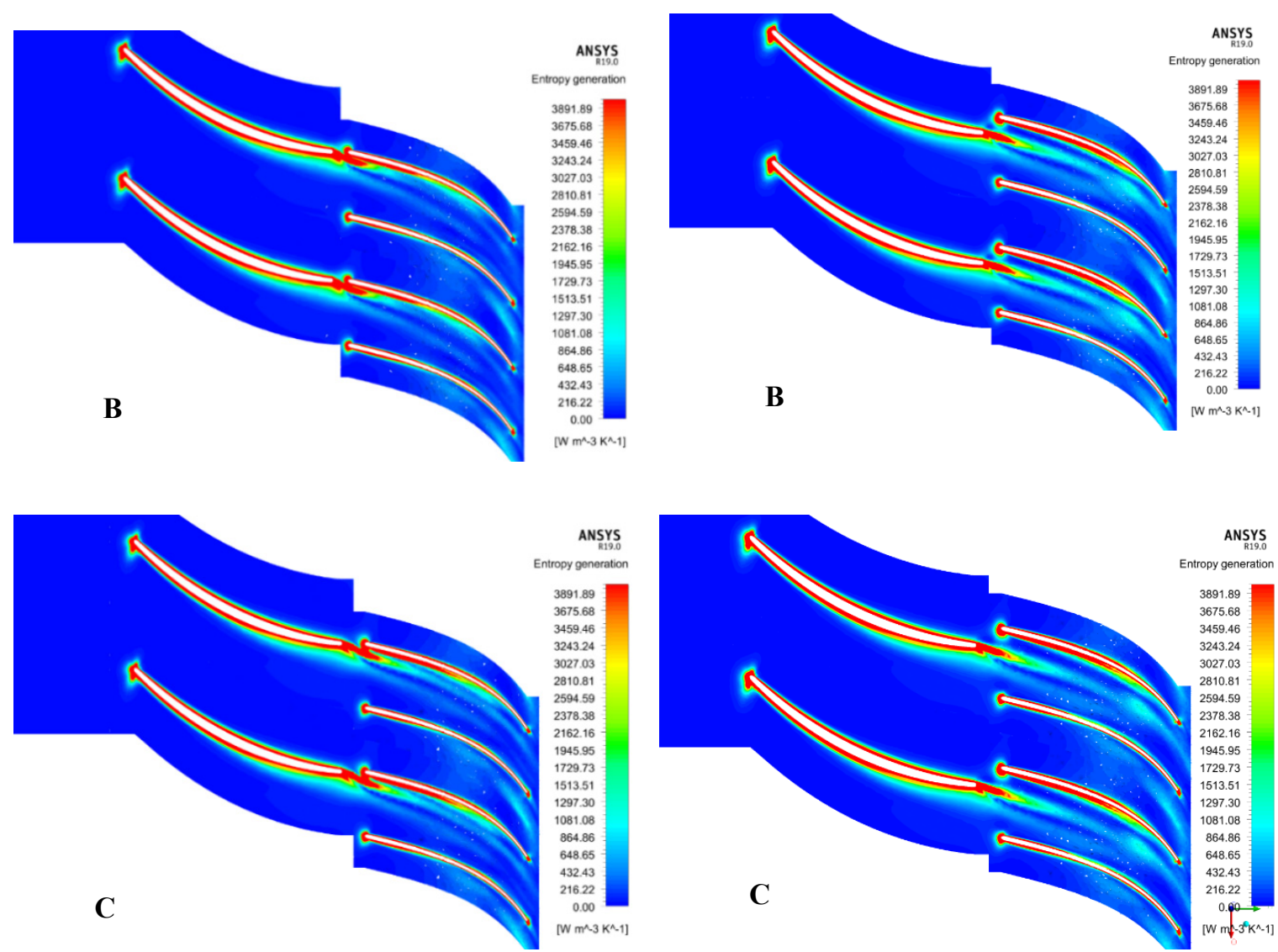

(a). Entropy generation map at 50\% span, $0 \%$ clock. Axial gap $0.5 \mathrm{~mm}(\mathrm{~A}), 1 \mathrm{~mm}$ (B) and $1.5 \mathrm{~mm}(\mathrm{C}) . \mathrm{mr}=8.3$

(b). Entropy generation map at 50\% span, $25 \%$ clock. Axial gap $0.5 \mathrm{~mm}, \mathrm{mr}=8.1(\mathrm{~A})$; $1 \mathrm{~mm}, \mathrm{mr}=8.2(\mathrm{~B}) ; 1.5 \mathrm{~mm}, \mathrm{mr}=8.3(\mathrm{C})$

Figure 17. Entropy generation maps of tandem B configuration

Figure 18 reports the same map for the "optimal" configuration (Tandem B): here, the inducer wake energizes the BL on the pressure side of the exducer, flattening on the high pressure flow on the pressure side of the exducer and providing a cleaner outlet. Above $50 \%$ clock, the blade interaction does not contribute significantly to the Mach losses.

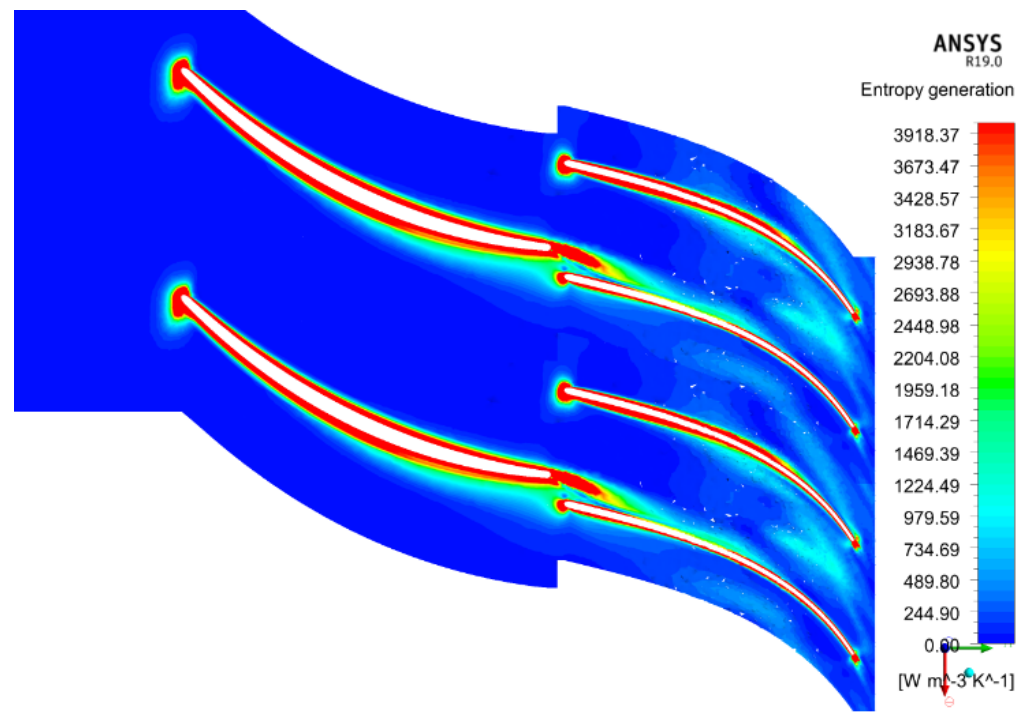

Figure 18. Entropy generation map at $50 \%$ span, $75 \%$ clock, $0.5 \mathrm{~mm}$ gap configuration, $\mathrm{mr}=8.2$ (lb/min). 


\section{Conclusions}

A tandem radial compressor for a hybrid compound turbocharger has been designed and simulated. The configuration has been improved via a DOE-guided series of refinements. The final geometry has a higher compression ratio than the original unit with an improved surge margin and a lower sensitivity to choking.

Funding: This research received no external funding

\section{References}

1. Hu, B.; Turner, J.W.G.; Akehurst, S.; Brace, C.; Copeland, C. Observations on and Potential Trends for Mechanically Supercharging a Downsized Passenger Car Engine: A Review. Proc. Inst. Mech. Eng. Part D J. Automob. Eng. 2017, 231, 435-456.

2. Patil, C.; Sanjyot, V.; Swapnil, W. A Review of Engine Downsizing and Its Effects. Int. J. Curr. Eng. Technol. 2017, 7, 319-324.

3. Capata, R. New Power Train Concept for a City Hybrid Vehicle. In Proceedings of the WEF-1 International Conference, Roma, Italy, 14 September-5 October 2020.

4. Muqeem, M.; Manoj, K. Turbocharging of IC engines: A review. Int. J. Mech. Eng. Technol. IJMET 2013, 4, 142-149.

5. Heywood, J.B. Internal Combustion Engine Fundamentals; McGraw-Hill Education: New York City, NY, USA, 2016.

6. Erdmenger, R.R.; Michelassi, V. Influence of Tandem Inducers on the Performance of High Pressure Ratio Centrifugal Compressors, ASME Paper GT2015-43001. In Proceedings of the ASME Turbo Expo 2015: Turbine Technical Conference and Exposition Proceedings, Montreal, QC, Canada, 15-19 June 2015.

7. Hanus, D.; Censky, T.; Neveceral, J.; Horky, V. First stage of the centrifugal compressor design with tandem rotor blades. In Proceedings of the ISABE-17th International Symposium on Airbreathing Engines, Munich, Germany, 4-9 September 2005; Paper 1161.

8. Hlavá, D.; Hanus, D. Results of the Development of a Tandem-Bladed Centrifugal Compressor Stage. Studentská Tvůrčí Činnost, 2016. Available online: http://stc.fs.cvut.cz/pdf16/6520.pdf (accessed on 1 March 2019).

9. Qureshi, S.R.; El-Leathy, A.; Ud-Din, K.S.; Umer, U.; Chaochen, M.; Danish, S.N. Numerical Investigation and Comparison of a Tandem-Bladed Turbocharger Centrifugal Compressor Stage with Conventional Design. Int. J. Therm. Sci. 2014, 23, 523-534.

10. Silvestri, T. CFD Analysis of a Radial Turbine Stage with Variable NGV Control. Master's Thesis, University of Roma Sapienza, Roma, Italy, 2020.

11. Cuturi, N. CFD Analysis of a Radial Compressor Stage with Inducer Blades. Master's Thesis, University of Roma Sapienza, Roma, Italy, 2019.

12. Mohtar, H. Increasing Surge Margin of Turbocharger Centrifugal Compressor Automotive. Ph.D. Thesis, Ecole Centrale de Nantes, Nantes, France, 2010.

13. Bejan, A. Entropy Generation through Heat and Fluid Flow, 1st ed.; J. Wiley \& Sons, Inc.: Hoboken, NJ, USA, 1982.

(C) 2020 by the authors. Licensee MDPI, Basel, Switzerland. This article is an open access article distributed under the terms and conditions of the Creative Commons Attribution (CC BY) license (http://creativecommons.org/licenses/by/4.0/). 